\title{
Gravity Local Search Inspired Particle Swarm Algorithm for Economic Power Dispatch Planning Problem in Small Scale System
}

\author{
Navpreet Singh Tung ${ }^{1}$, Sandeep Chakravorty ${ }^{2}$ and Harkamal Singh Bhullar ${ }^{3}$ \\ ${ }^{1}$ Assistant Professor, Department of Electrical Engineering, \\ Bhutta Group of Institutions, Ludhiana, India \\ ${ }^{2}$ Dean and Professor, Department of Electrical Engineering, \\ Baddi University, Baddi, India \\ ${ }^{3}$ Assistant Professor, Department of Electrical Engineering Baba Kuma Singh \\ Engineering College,Amritsar,India \\ ${ }^{1}$ icenitj@gmail.com \\ ${ }^{2}$ sandeep@baddiuniv.ac.in
}

\begin{abstract}
This research presents novel Particle swarm optimization inspired by gravitational based search method to solve active power dispatch problem in electrical power system planning. The proposed PSO utilizes the operator of social thinking coupled with search capacity of gravity inspired algorithm to formulate and develop technique for active power dispatch problem to satisfy power demand requirements. Optimal scheduling of generators and system constraints to match load demand and losses is successfully done with proposed method. Total operating cost is minimized satisfying various bounds of system with proposed method. Exploration and convergence efficiency are evaluated to checklist the computational efficiency and robustness of the proposed technique. The suggested technique is tested and evaluated on different test systems comprises three, five, six test systems. Test results are compared with other techniques presented in literature .Investigations shows promising results which further benchmark the effectiveness of proposed method to solve complex optimization non linear problems.
\end{abstract}

Keywords: Unit Commitment (UC), Economic Power Dispatch (EPD), Gravity Local Search Particle Swarm Algorithm (GLSPSA)

\section{Introduction}

chnique for multi area APD considering tie line constraints Dr.T.Govindaraj[15] and PrThe APD problem is one of the core area of today's power system. The objective of the APD is to find the efficient generation among the genera units, so that the total fuel cost is minimized while matching the power balance equations and various other constraints in the system. Different evolutionary algorithms and swarm intelligence techniques have been successfully applied to APD problem. Duman S. et al[1] fixed APD problem with gravitational search algorithm considering the impact of valve point .Wood and Zhu [2,3] presents different aspects of economic dispatch and optimization. Navpreet Singh Tung and Sandeep Chakravorty[4,5] proposed pattern search(PS) and differential evolution(DE) based solution to APD problem considering losses. Hamid Boujeboudja [6] presented real coded genetic algorithm for APD problem. Vikram Kamboj[7] presents formulation of Multi Area UC problem .J Mary[8] applied shuffled leap grog technique for UC and APD problem on different test cases.Vikram Singh[9] et al developed PSO technique for short term APD and UC problem considering emission impact.V.K Kamboj[10] successfully formulated $\mathrm{BBO}$ for $\mathrm{UC}$ and $\mathrm{ED}$ problem.Adriane[11] coined cuckoo search(CS) for AD 
problem and different test cases were presented. A. A. Mousa[13] presented hybrid evolutionary and local search method for APD problem with emission constraint. Manisha Sharma[14] applied evolutionary technique for multi area APD considering tie line constraints Dr.T.Govindaraj[15] and Priyanka Roy[16] proposed genetic algorithm and modified shuffled frog leaping for APD problem.

\section{Problem Design}

The EPD problem [2, 7, and 10]] may be expressed by minimizing the operating fuel cost of generator units subjected to various constraints. As the load demand is variable, the power generation has to be adjusted to match the balance between loads and generation of a power system. The EPD model consists of $n$ generating units online.

The EPD problem can be expressed as.

\section{A. Fuel Cost Model}

$\mathrm{C}\left(\mathrm{P}_{\mathrm{Gi}}\right)=\left(\mathrm{a}_{\mathrm{i}} * \mathrm{P}_{\mathrm{Gi}}{ }^{2}+\mathrm{bi}^{*} * \mathrm{P}_{\mathrm{Gi}}+\mathrm{c}_{\mathrm{i}}\right)$ Rs where $\mathrm{i}=1 . . \mathrm{N}(\mathrm{a}, \mathrm{b}, \mathrm{c}$ are cost co-efficients $)$

B. Power Balance Constraints

$\mathrm{P}_{\mathrm{Gi}}-\mathrm{PD}-\mathrm{PL}=0 \mathrm{P}_{\mathrm{Gi}, \min } \leq \mathrm{P}_{\mathrm{Gi}} \leq \mathrm{P}_{\mathrm{Gi}, \max }$ where $\mathrm{i}=1,2 \ldots \ldots \mathrm{N}($ Limits of Power Generation $)$

\section{Transmission Losses Formulation}

$P_{L}=\sum_{i=1}^{N} \sum_{j=1}^{N} P_{G i} B_{i j} P_{G j}+\sum_{i=1}^{N} B_{0 i} P_{G i}+B_{00}$

(B- Loss coefficients)

\section{Gravity Local Search Particle Swarm Optimization}

The inspiration for the hybridization[12] of GLSPSA is to couple the capability of social thinking factor in PSO magnetize with the local search power of Gravity based local search algorithm. This technique accepts the agents as objects and the position of ith agent is given by-

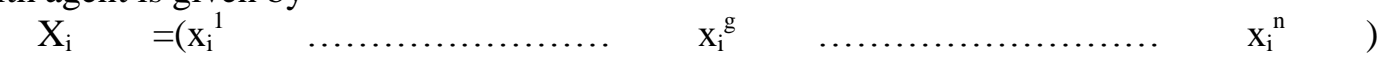

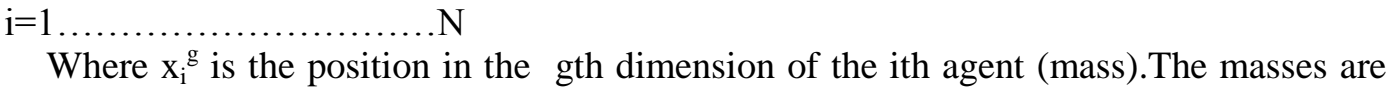
presented randomly and the force acting on mass $i$ from mass $j$ is given as-

$$
\mathrm{F}_{\mathrm{jid}}=\mathrm{G}(\mathrm{t}) *\left(\mathrm{M}_{\mathrm{i}}(\mathrm{t}) * \mathrm{M}_{\mathrm{j}}(\mathrm{t})\right) / \mathrm{R}_{\mathrm{ij}}+\mathrm{c} *\left(\mathrm{X}_{\mathrm{jd}}(\mathrm{t})-\mathrm{X}_{\mathrm{id}}(\mathrm{t})\right)
$$

Where $M_{i}(t)$ and $M_{j}(t)$ are masses of objects $i$ and $j, G(t)$ is the gravitational constant at time $t, c$ is a small constant, $R_{i j}(t)$ is the Euclidean distance between $i$ and $j$ objects.

(5)

$$
R_{\mathrm{ij}}(\mathrm{t})=\left\|\mathrm{X}_{\mathrm{i}}(\mathrm{t}), \mathrm{X}_{\mathrm{j}}(\mathrm{t})\right\| 2
$$

Gravitational constant $\mathrm{G}(\mathrm{t})$ is initialized in random fashion in the starting and is decremented over the period time to control and effect the search accuracy precision.

$$
\mathrm{G}(\mathrm{t})=\mathrm{G}_{\mathrm{o}} \mathrm{e} \propto \mathrm{t} / \mathrm{T}
$$

(6)

It shows $\mathrm{G}$ is the function of time $t$ and initial value $\mathrm{G}_{0}$, where $\mathrm{G} 0$ is the initial value of gravitational constant, $\alpha$ is the user constant and $\mathrm{T}$ is the maximum number of iterations and $\mathrm{t}$ is the current iteration.

Assume the total force acting on agent $i$ in the dimension $d$ is presented as-

$F_{i t}(t)=\sum_{j}=\operatorname{rand}_{i} F_{j i d}(t)$ 
Where, randj is a random number between the interval $[0,1]$.

The acceleration of ith agent at iteration $t$ having d dimension is given by the law of motion-

$\alpha \mathrm{c}_{\mathrm{id}}(\mathrm{t})=\operatorname{Fid}(\mathrm{t}) / \mathrm{M}_{\mathrm{i}}(\mathrm{t})$

The velocity of an agent is calculated as-

$V_{\text {id }}(t+1)=w \cdot V_{\text {id }}(t) c 1 \times \operatorname{rand} \times c_{\text {idd }}(t)+c 2 \times \operatorname{rand} \times\left(\right.$ gbest $\left.-\mathrm{Xid}_{\text {id }}(t)\right)$

Where $\operatorname{vid}(t)$ is the velocity of agent $i$ at iteration $t$ in dimension $d, \mathrm{cj}^{\prime}$ is a weighting factor, $\mathrm{w}$ is a weighting function, rand is a random number between 0 and $1, \alpha \operatorname{cid}(\mathrm{t})$ is the acceleration of ith agent at iteration $t$ in dimension $d$ and gbest is the best solution found till now.

At each, iteration the position of an agent is calculated as-

$\operatorname{Xid}(\mathrm{t}+1)=\operatorname{Xid}(\mathrm{t})+\operatorname{Vid}(\mathrm{t}+1)$

Where $\operatorname{vid}(t+1)$ is the velocity of next agent and xid is the position of ith agent in dth dimension at iteration $t$. The value of masses of agents are calculated by comparison of fitness-

$$
\begin{gathered}
\mathrm{mi}_{\mathrm{i}}(\mathrm{t})=\text { currentfit nessi }(\mathrm{t})-0.99 \times \operatorname{worst}(\mathrm{t}) /(\operatorname{best}(\mathrm{t})-\operatorname{worst}(\mathrm{t})) \\
\mathrm{i}=1 \ldots \ldots \ldots \ldots \ldots \ldots \ldots \ldots \ldots \ldots \ldots \ldots \ldots \ldots \ldots \ldots \\
M_{i}(\mathrm{t})=\mathrm{m}_{\mathrm{i}}(\mathrm{t}) * 5 / \sum_{\mathrm{j}}=\mathrm{nnm} \mathrm{m}(\mathrm{t})
\end{gathered}
$$

Where current-fitnessi ( $t$ ) is the fitness value of the agent $i$ at any time $t$, and best (t) and worst ( $\mathrm{t}$ ) are the minimum and maximum fitness value of all agents. The agents browsing in the search space are attracted towards other agents by means of gravity force and pushes a run to the agents having heavier mass. The heavier mass represents a good solution. Here gbest assists them in finding the global optima. The optimal solution is found by using the exploitation ability of PSO. Global search and local search trade-off is done by contrasting the values $\mathrm{C} 1$ and $\mathrm{C} 2$. 


\section{- Design and Formulation of GLSPSA}

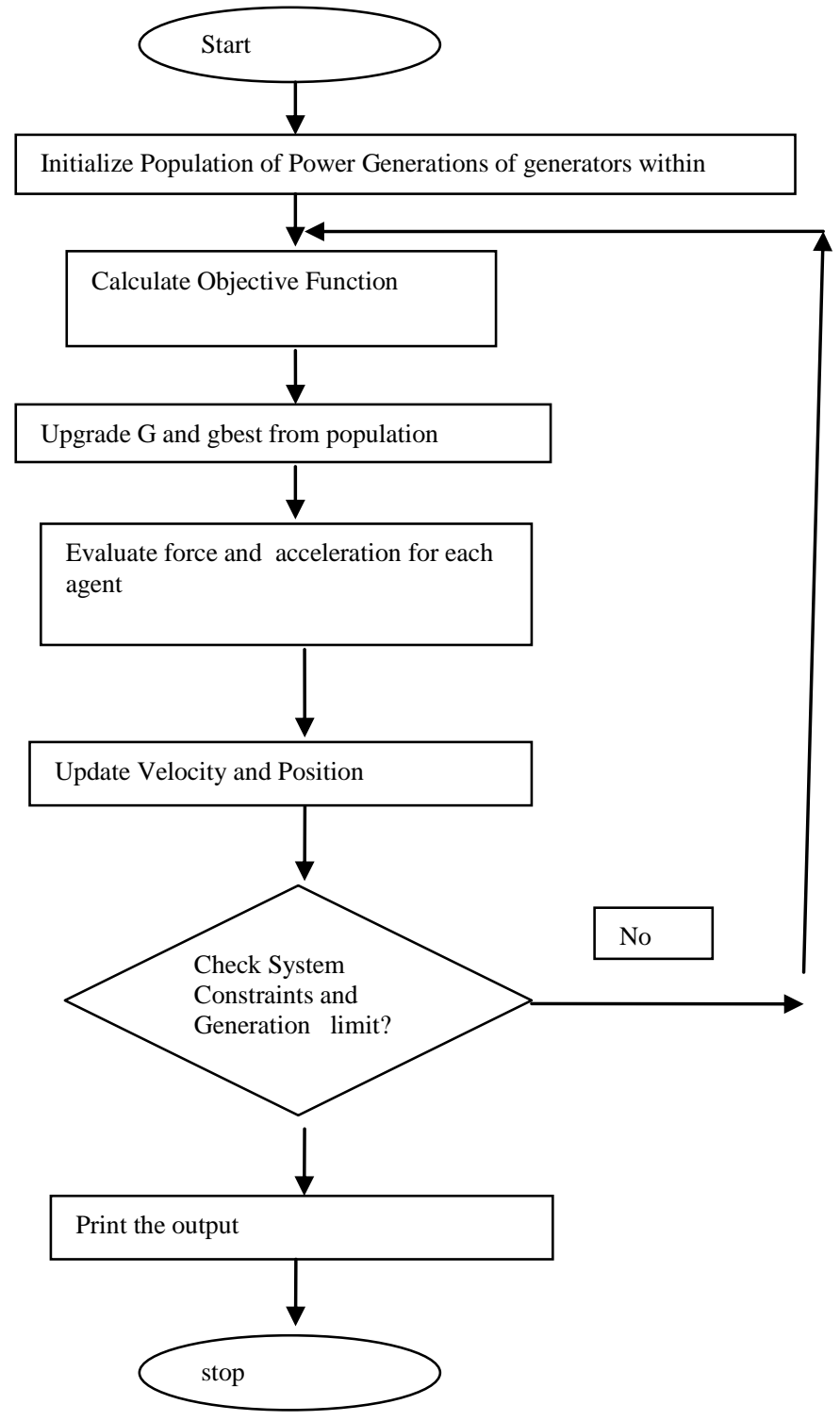

\section{- Simulation Flow}

\section{Step1: Feasible Boundary location}

Agents are randomly initialized and located between the minimum and maximum operating limits of generators. Each agent should match the system constraints.

\section{Step 2: Objective Function}

This evaluates for each agent while constraints are satisfied. Update $\mathrm{G}$ and gbest for the population.

\section{Step 3: Force}

Total force acting on agent $\mathrm{i}$ in different dimensions is evaluated.

\section{Step 4: Mass and Acceleration of an agent}

The acceleration of ith agent in d dimension is solved and mass is calculated.

Step 5: Update position and velocity of every agent

The next velocity of agent is calculated and position is updated

Step 6: Finishing criteria

Repeat process 2 to 5 until maximum number of iterations is reached. 


\section{Comparative Algorithm Numerical Setting}

Different algorithms settings have been shown below. They have been taken after number of runs [11].Test results are presented with these settings.

- Shuffled Frog Leaping Algorithm (SFLA): (meme- plexes) $=10,($ frogs $)=10$, $($ submemeplexes $)=2$, Smax $($ step $)=5,($ evolutions $)=5$.

- Particle Swarm Optimization (PSO): Population $=20, \mathrm{c} 1=2, \mathrm{c} 2=2$.

- Bacterial Foraging Optimization $(\mathrm{BFO})$ : $($ bacteria $)=20, \mathrm{Nc}=10, \mathrm{Ns}=4$, Nre $=4$, Ned $=$ cycles, ped $=0.25$, dattract $=0.1$, wattract $=0.2$, hrepellant $=0.1$, wrepellant $=$ 10 . .

- Harmony Search (HS): (harmony memory size) $=2$, (harmony memory considering rate $)=80$, (pitch adjusting rate $)=0.4$.

- Firefly Algorithm (FA): $\mathrm{n}$ (fireflies) $=20, \alpha$ (randomization factor) $=0.2, \beta 0$ $($ attractiveness $)=1.0, \Omega$ (absorption coefficient $)=1.0$.

- Artificial Bee Colony $(\mathrm{ABC}):($ colony $)=20, \mathrm{BN}($ employed bees $)=10,($ onlookers $)=$ $10, \mathrm{Cmax}=100$

- Cuckoo Search (CS): (nests) $=20$, (discovery rate $)=0.25$.

- Gravitational Search Algorithm(GSA): Iterations $\mathrm{T}=100, \alpha=8$, Gravitational Constant $\mathrm{G} 0=2$

- Gravity Local Search Particle Swarm Algorithm: Population size $n=1000$, acceleration coefficient $\alpha=8$, Gravitational Constant $\mathrm{G} 0=1$, weight $\mathrm{C} 1=2.5, \mathrm{C} 2=1.8$

- Different algorithms settings have been shown below. They have been taken after number of runs[11].Test results are presented with these settings.

- Shuffled Frog Leaping Algorithm (SFLA): (meme- plexes) $=10$, (frogs) $=10$, $($ submemeplexes $)=2$, Smax $($ step $)=5,($ evolutions $)=5$.

- Particle Swarm Optimization (PSO): Population $=20, \mathrm{c} 1=2, \mathrm{c} 2=2$.

- Bacterial Foraging Optimization $(\mathrm{BFO}):($ bacteria $)=20, \mathrm{Nc}=10, \mathrm{Ns}=4$, Nre $=4$, $\mathrm{Ned}=$ cycles, ped $=0.25$, dattract $=0.1$, wattract $=0.2$, hrepellant $=0.1$, wrepellant $=$ 10 .

- Harmony Search (HS): (harmony memory size) $=2$, (harmony memory considering rate $)=80$, (pitch adjusting rate $)=0.4$.

- Firefly Algorithm (FA): $\mathrm{n}$ (fireflies) $=20, \alpha$ (randomization factor) $=0.2, \beta 0$ $($ attractiveness $)=1.0, \Omega$ (absorption coefficient $)=1.0$.

- Artificial Bee Colony $(\mathrm{ABC}):($ colony $)=20, \mathrm{BN}($ employed bees $)=10,($ onlookers $)=$ $10, \mathrm{Cmax}=100$

- Cuckoo Search (CS): (nests) $=20,($ discovery rate $)=0.25$.

- Gravitational Search Algorithm(GSA): Iterations $T=100, \alpha=8$, Gravitational Constant $\mathrm{G} 0=2$

- Gravity Local Search Particle Swarm Algorithm: Population size $n=1000$, acceleration coefficient $\alpha=8$, Gravitational Constant $\mathrm{G} 0=1$, weight $\mathrm{C} 1=2.5, \mathrm{C} 2=1.8$

\section{EPD Formulation using GLSPSO}

Variables

$>$ Power Generation (PG) and cost coefficients (a,b,c) of units with objective function as fuel cost, quadratic in nature. Power Generation variable should be initialized as starting point for initial solution in GLSPSA.

$>$ Constraints

$>$ Equality Constraints: Power Generation-Power Demand-Power losses $=0\left(\mathrm{P}_{\mathrm{G}}-\mathrm{P}_{\mathrm{d}}-\mathrm{P}_{\mathrm{L}}\right)$

$>$ In-Equality Constraints: Power Generation should be between minimum and maximum limit of power generation.

$>$ Variables in constraints should be incorporated in GLSPSA.

$>$ Stopping Criteria 
It is the maximum generation limit for optimum solution.

\section{Test Systems}

GLSPSA has been tested to solve the APD problems in three different test cases[4,5,6,11] for investigating its optimization power, computational efficiency subjected to power generation ranges of the generating units and transmission losses are incorporated. We presented the test systems, the algorithm settings and the numerical results as follows.

\subsection{Three-Generating Unit System [11]}

This case study incorporates three generating units. The coefficients of fuel cost and the limits of the generation units.

\subsection{Six-Generating Unit System [11]}

\begin{tabular}{|l|c|c|c|c|c|}
\hline Unit & $\begin{array}{l}\mathrm{a}\left(\$ / \mathrm{MW}_{2}\right. \\
)\end{array}$ & $\mathrm{b}(\$ / \mathrm{MW})$ & $\begin{array}{l}\mathrm{c}(\$ \\
)\end{array}$ & $\mathrm{PG}_{\min }(\mathrm{MW})$ & $\mathrm{PG}_{\max }(\mathrm{MW})$ \\
\hline 1 & 0.008 & 7 & 200 & 10 & 85 \\
\hline 2 & 0.009 & 6.3 & 180 & 10 & 80 \\
\hline 3 & 0.007 & 6.8 & 140 & 10 & 70 \\
\hline
\end{tabular}

This case study incorporates six generating units. The coefficients of fuel cost and the limits of the generation units.

\begin{tabular}{|l|c|c|c|c|c|}
\hline Unit & $\begin{array}{l}\mathrm{a}\left(\$ / \mathrm{MW}_{2}\right. \\
)\end{array}$ & $\mathrm{b}(\$ / \mathrm{MW})$ & $\begin{array}{l}\mathrm{c}(\$ \\
)\end{array}$ & $\mathrm{PG}_{\min }(\mathrm{MW})$ & $\mathrm{PG}_{\max }(\mathrm{MW})$ \\
\hline 1 & 0.007 & 7 & 240 & 100 & 500 \\
\hline 2 & 0.005 & 10 & 200 & 50 & 200 \\
\hline 3 & 0.009 & 8.5 & 220 & 80 & 300 \\
\hline 4 & 0.009 & 11 & 200 & 50 & 150 \\
\hline 5 & 0.0080 & 10.5 & 220 & 50 & 200 \\
\hline 6 & 0.0075 & 12 & 120 & 50 & 120 \\
\hline
\end{tabular}

\subsection{IEEE 25 Bus System with Five Generating Units System $[4,5,6]$}

This case study incorporates five generating units .The coefficients of fuel cost and the capacities of the generation units.

\begin{tabular}{|l|c|c|c|c|c|}
\hline Unit & $\begin{array}{l}\mathrm{a}(\$ / M W 2 \\
\text { ( }\end{array}$ & $\begin{array}{l}\mathrm{b}(\$ / \mathrm{MW}) \\
\mathrm{c}(\$ \\
\mathrm{PG}_{\min }(\mathrm{MW})\end{array}$ & $\mathrm{PG}_{\max }(\mathrm{MW})$ \\
\hline 1 & 0.0015 & 1.8 & 40 & 100 & 300 \\
\hline 2 & 0.0030 & 1.7 & 60 & 80 & 150 \\
\hline 3 & 0.0012 & 2.1 & 100 & 80 & 200 \\
\hline 4 & 0.0080 & 2.0 & 25 & 20 & 100 \\
\hline 5 & 0.0010 & 1.9 & 120 & 100 & 300 \\
\hline
\end{tabular}




\section{Results and discussions}

\section{A. Layout}

- Test system comprises of with 3 units, 5 units and 6 units system for variable power demand.

- Optimal Power generation (MW) for every test system has been carried out using GLSPSA

- Comparison of Total Operating Cost, CPU Computational time, Best Cost, Worst Cost, Mean Cost, Standard Deviation(SD) has been presented for different test cases.

\section{B. Simulation and Numerical Result}

A complete investigation and computation were done on proposed technique. Comparative Analysis has been carried out with other swarm techniques. Table 1.showcase the optimum power generation for every unit, total operating cost and CPU computational time using proposed GLSPSA technique on IEEE 25 bus system. In table 2, comparative analysis has been presented with other techniques like pattern search(PS), differential evolution(DE), real and binary coded genetic algorithm(RCGA,BCGA,BFGS) in terms of cost, CPU computational time. In Table ,3 an investigation states that proposed technique GLSPSA is outperformed other techniques as total cost and computational time turned out to be less as compared to other techniques in literature PS, DE, RCGA, BCGA, BFGS. Test results shows the computational efficiency, problem solution quality of proposed technique as compared to other techniques.

Table 1. Power Generation, Total Cost and Computational Time using GLSPSA on IEEE 25 Bus System

\begin{tabular}{|l|l|l|l|l|l|l|}
\hline $\begin{array}{l}\text { PG1 } \\
(\mathrm{MW})\end{array}$ & $\begin{array}{l}\text { PG2 } \\
(\mathrm{MW})\end{array}$ & $\begin{array}{l}\text { PG3 } \\
(\mathrm{MW})\end{array}$ & $\begin{array}{l}\text { PG4 } \\
(\mathrm{MW})\end{array}$ & $\begin{array}{l}\text { PG5 } \\
(\mathrm{MW})\end{array}$ & $\begin{array}{l}\text { Cost } \\
(\$ / \mathrm{hr})\end{array}$ & $\begin{array}{l}\text { Time } \\
(\mathrm{Sec})\end{array}$ \\
\hline 181 & 131.41 & 113.36 & 70.5 & 263.65 & 2000.0239 & 2 \\
\hline
\end{tabular}

Table 2.Results Comparison with other Techniques $[4,5,6]$ on IEEE 25 Bus System

\begin{tabular}{|l|l|l|l|l|l|l|}
\hline Parameters & GLSPSA & PS & DE & RCGAs & BCGAs & BFGS \\
\hline $\begin{array}{l}\text { PG1 } \\
\text { (MW) }\end{array}$ & $\mathbf{1 8 1 . 8 8}$ & 212.244 & $\begin{array}{l}212.244 \\
1\end{array}$ & 213.68 & 206.72 & 211.30 \\
\hline $\begin{array}{l}\text { PG2 } \\
\text { (MW) }\end{array}$ & $\mathbf{1 3 1 . 5 1}$ & 122.789 & $\begin{array}{l}122.78 \\
87\end{array}$ & 127.46 & 121.64 & 126.30 \\
\hline $\begin{array}{l}\text { PG3 } \\
\text { (MW) }\end{array}$ & $\mathbf{1 1 3 . 3 8 8}$ & 140.305 & $\begin{array}{l}140.305 \\
2\end{array}$ & 141.93 & 151.82 & 151.29 \\
\hline $\begin{array}{l}\text { PG4 } \\
\text { (MW) }\end{array}$ & $\mathbf{7 0 . 5 8}$ & 27.296 & 27.2958 & 29.53 & 33.21 & 71.24 \\
\hline $\begin{array}{l}\text { PG5 } \\
\text { (MW) }\end{array}$ & $\mathbf{2 6 3 . 6 5 5}$ & 268.366 & $\begin{array}{l}268.366 \\
2\end{array}$ & 258.86 & 258.05 & 211.31 \\
\hline $\begin{array}{l}\text { Cost } \\
(\$ / \text { hr })\end{array}$ & $\mathbf{2 0 0 7 . 0 3 9}$ & 2009.312 & 2009.3145 & 2010.8 & 2011.0 & 2029.3 \\
\hline $\begin{array}{l}\text { Time } \\
(\text { Sec) }\end{array}$ & $\mathbf{1 . 4 2}$ & 1.2 & 7 & 1.6 & 4.78 & 0.0 \\
\hline
\end{tabular}


Table 3. Computational Time and Total Cost Comparison with other Techniques $[4,5,6]$ on IEEE 25 Bus System

\begin{tabular}{|l|l|l|l|l|l|l|}
\hline Parameters & GLSPSA & PS & DE & RCGAs & BCGAs & BFGS \\
\hline $\begin{array}{l}\text { Cost } \\
(\$ \mathrm{hr})\end{array}$ & $\mathbf{2 0 0 0 . 0 3 9}$ & 2009.312 & 2009.3145 & 2010.8 & 2011.0 & 2029.3 \\
\hline $\begin{array}{l}\text { Time } \\
(\text { Sec })\end{array}$ & $\mathbf{1 . 4}$ & 1.2 & 7 & 1.6 & 4.78 & 0.0 \\
\hline
\end{tabular}

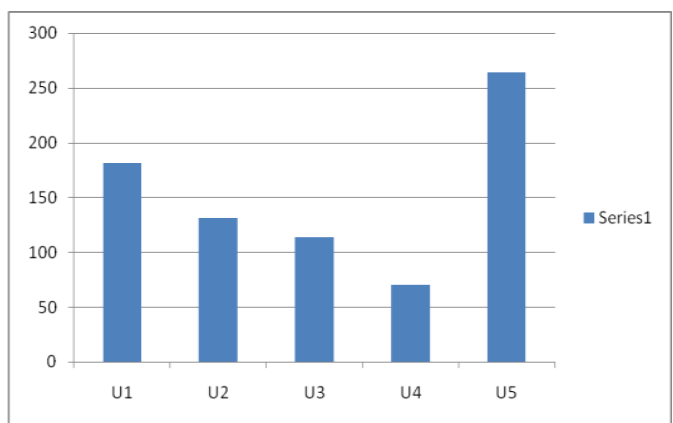

Figure 1. Power Generation using GLSPSA on IEEE 25 Bus System

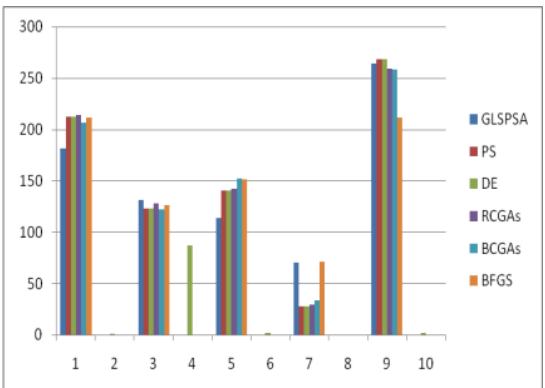

Figure 2. Power Generation Comparison on IEEE 25 Bus System

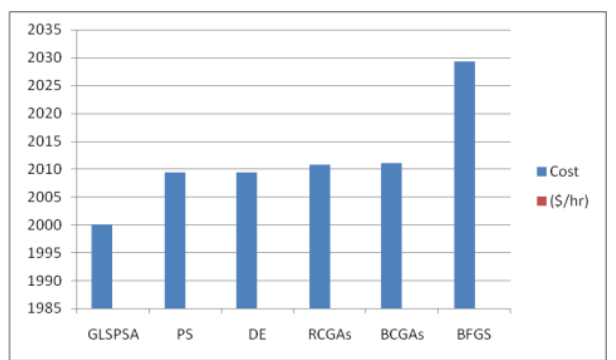

Figure 3. Comparison of Total Cost on IEEE 25 Bus System

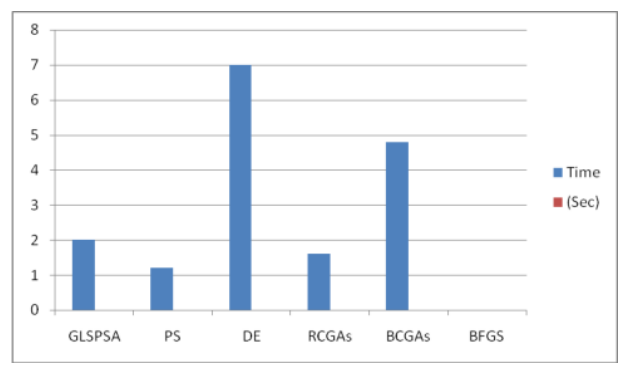

Figure 4. Comparison of CPU Computational Time on IEEE 25 Bus System 


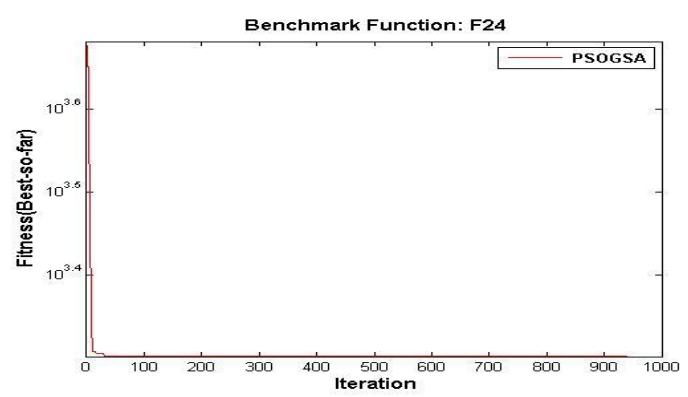

\section{Figure 5. Convergence of Solution on IEEE 25 Bus System}

Figure 1 and 2 represents power generation and comparison of test system of IEEE 25 bus system. Figure 3 and 4 shows cost and computational time comparative analysis. Figure 5. shows convergence of solution with iterations with GLSPSA.

Table 4 tabulates the result of power Generation on six unit system and total operating cost to run these units. Table 5 shows the comparative analysis of power generation, cost of proposed technique GLSPSA with literature techniques like cuckoo search(CS),Artificial Bee colony(ABC),Firefly Algorithm(FA),Gravitational Search Algorithm(GSA).Table 6 stored the cost numerical results and comparison was made with other techniques cuckoo search(CS),Artificial Bee colony(ABC),Firefly Algorithm(FA),Gravitational Search Algorithm(GSA),Harmony search(HS),Particle Swarm Optimization(PSO),Shuffled frog leaping algorithm(SFLA) ,Bacterial foraging optimization(BFO).Test results shows that cost using GLSPSA is less as compared to other techniques PSO, ABC, SFLA, CS, HS, BFO, GSA. Thus, proposed approach is more robust and computationally efficient as compared to other techniques. Figure 6 shows convergence of solution for proposed method GLSPSA with iterations.

Table 7 latched numerical parameters to showcase the convergence power, quality of solutions and efficiency of computational power. These are converted and presented in terms of parameters Standard deviation, mean cost, best cost, worst cost and comparative investigation has been carried out with other techniques PSO, ABC, SFLA, CS, HS, BFO, GSA,FA. Standard Deviation for proposed technique is minimum among other techniques. Best cost, Worst cost, Mean cost are minimum with iterations as checked with other methods.These investigations benchmarks the robustness, reliability, global minima convergence, solution efficiency, computation power of proposed GLSPSA technique as compared to others. To concrete the result, further investigation is done on small system having three unit system. Table 8 and 9 substantiate the upper investigations. Figure 6 shows the convergence of solution with iterations. Proposed technique GLSPSA searches for best solution among initial population of generated power within bounds. GLSPSA is successfully applied to different set of systems. Test results show the promise of proposed technique. In addition, the results substantiate the robustness, precise convergence and efficiency of this optimization algorithm. The main advantage of GLSPSA is a core ability for finding the global solution from among local minima values. From the results, it can be infer that GLSPSA is a competitive technique for solving complex non smooth optimization problems in EPD.

\section{Table 4. Power Generation, Total Cost and Computational Time using} GLSPSA on Six Unit System [11]

\begin{tabular}{|l|l|l|l|l|l|l|}
\hline $\begin{array}{l}\text { PG1 } \\
\text { (MW) }\end{array}$ & $\begin{array}{l}\text { PG2 } \\
\text { (MW) }\end{array}$ & $\begin{array}{l}\text { PG3 } \\
\text { (MW) }\end{array}$ & $\begin{array}{l}\text { PG4 } \\
\text { (MW) }\end{array}$ & $\begin{array}{l}\text { PG5 } \\
\text { (MW) }\end{array}$ & $\begin{array}{l}\text { PG6 } \\
\text { (MW) }\end{array}$ & $\begin{array}{l}\text { Cost } \\
(\$ / h r)\end{array}$ \\
\hline $\mathbf{1 5 4 . 4 4 3 4}$ & $\mathbf{5 0 . 0 0 0 0}$ & $\mathbf{2 5 6 . 8 3 3 8}$ & $\mathbf{1 1 1 . 7 3 1 8}$ & $\mathbf{6 1 . 9 6 8 1}$ & $\mathbf{7 6 . 0 2 2 9}$ & $\mathbf{7 8 9 1 . 1}$ \\
\hline
\end{tabular}


Table 5.Results Comparison with other Techniques on Six Unit System [11]

\begin{tabular}{|l|l|l|l|l|l|}
\hline Parameters & GLSPSA & CS & ABC & FA & GSA \\
\hline $\begin{array}{l}\text { PG1 } \\
\text { (MW) }\end{array}$ & $\mathbf{1 5 4 . 4 4 3 4}$ & 324.113 & 323.043 & 293.312 & 298.44 \\
\hline $\begin{array}{l}\text { PG2 } \\
\text { (MW) }\end{array}$ & $\mathbf{5 0 . 0 0 0 0}$ & 76.859 & 54.965 & 79.546 & 75.67 \\
\hline $\begin{array}{l}\text { PG3 } \\
\text { (MW) }\end{array}$ & $\mathbf{2 5 6 . 8 3 3 8}$ & 158.094 & 147.354 & 123.334 & 132.46 \\
\hline $\begin{array}{l}\text { PG4 } \\
\text { (MW) }\end{array}$ & $\mathbf{1 1 1 . 7 3 1 8}$ & 50.000 & 50.000 & 69.700 & 58.73 \\
\hline $\begin{array}{l}\text { PG5 } \\
\text { (MW) }\end{array}$ & $\mathbf{6 1 . 9 6 8 1}$ & 51.963 & 85.815 & 79.546 & 76.89 \\
\hline $\begin{array}{l}\text { PG6 } \\
\text { (MW) }\end{array}$ & $\mathbf{7 6 . 0 2 2 9}$ & 50.000 & 50.233 & 63.778 & 56.78 \\
\hline $\begin{array}{l}\text { Cost } \\
\text { \$/hr) }\end{array}$ & $\mathbf{7 8 9 1 . 1}$ & 8356.06 & 8372.27 & 8388.45 & 8394.46 \\
\hline
\end{tabular}

Table 6. Results Comparison of Cost with other Techniques on Six Unit System [11]

\begin{tabular}{|l|l|l|l|l|l|l|l|l|l|}
\hline $\begin{array}{l}\text { Techni } \\
\text { que }\end{array}$ & $\begin{array}{l}\text { GLSP } \\
\text { SA }\end{array}$ & CS & ABC & FA & PSO & SFL & BFO & HS & GSA \\
\hline $\begin{array}{l}\text { Cost } \\
(\$ / h r)\end{array}$ & 7891.1 & 8356.0 & 8372.27 & 8388.4 & 8401 & 841 & 8428. & 8398. & 8394. \\
\hline
\end{tabular}

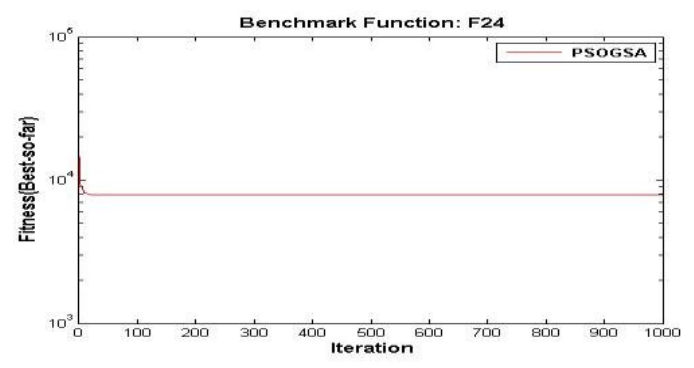

Figure 6. Convergence of Solution on Six Unit System

Table 7.Convergence, Computation and Exploration Capability Comparison with other Techniques on Six Unit System [11]

\begin{tabular}{|l|c|c|c|c|}
\hline Parameters & $\begin{array}{l}\text { Mean } \\
\text { Cost }(\$ / \mathrm{hr})\end{array}$ & $\begin{array}{l}\text { Standard } \\
\text { Deviation }\end{array}$ & $\begin{array}{l}\text { Worst } \\
\text { Cost }(\$ / \mathrm{hr})\end{array}$ & $\begin{array}{l}\text { Best } \\
\text { Cost }(\$ / \mathrm{hr})\end{array}$ \\
\hline CS & 8356.06 & 0.0008 & 8356.06 & 8356.06 \\
\hline ABC & 8457.16 & 57.726 & 8610.28 & 8372.27 \\
\hline FA & 8631.82 & 176.83 & 9082.00 & 8388.45 \\
\hline PSO & 8722.04 & 177.652 & 8912.16 & 8401.45 \\
\hline SFL & 8479.49 & 54.781 & 8604.29 & 8419.78 \\
\hline BFO & 8571.91 & 127.85 & 8909.85 & 8428.69 \\
\hline HS & 8541.72 & 99.531 & 8778.37 & 8398.06 \\
\hline GSA & 8567.89 & 78.99 & 8904.56 & 8394.46 \\
\hline GLSPSA & $\mathbf{7 8 9 1 . 1}$ & $\mathbf{0 . 0 0 0 0 9 7}$ & $\mathbf{7 8 9 1 . 1}$ & $\mathbf{7 8 9 1 . 1}$ \\
\hline
\end{tabular}


Table 8.Results Comparison with other Techniques on Three Unit System [11]

\begin{tabular}{|l|l|l|l|l|l|}
\hline Parameters & GLSPSA & CS & ABC & FA & GSA \\
\hline $\begin{array}{l}\text { PG1 } \\
\text { (MW) }\end{array}$ & $\mathbf{4 5 . 5 5}$ & 33.490 & 33.049 & 32.729 & 34.51 \\
\hline $\begin{array}{l}\text { PG2 } \\
\text { (MW) }\end{array}$ & $\mathbf{5 5 . 7 1}$ & 64.116 & 61.764 & 63.843 & 62.74 \\
\hline $\begin{array}{l}\text { PG3 } \\
\text { (MW) }\end{array}$ & $\mathbf{5 0 . 7 8}$ & 55.126 & 57.872 & 56.151 & 58.92 \\
\hline $\begin{array}{l}\text { Cost } \\
\text { (\$hr) }\end{array}$ & $\mathbf{1 5 4 2}$ & 1600.46 & 1600.51 & 1600.47 & 1600.56 \\
\hline
\end{tabular}

\section{Table 9. Convergence, Computation and Exploration Capability Comparison with other Techniques on Three Unit System [11]}

\begin{tabular}{|c|c|c|c|c|}
\hline Parameters & $\begin{array}{l}\text { Mean } \\
\text { Cost }(\$ / h r)\end{array}$ & $\begin{array}{l}\text { Standard } \\
\text { Deviation }\end{array}$ & $\begin{array}{l}\text { Worst } \\
\text { Cost }(\$ / h r)\end{array}$ & $\begin{array}{l}\text { Best } \\
\text { Cost }(\$ / h r)\end{array}$ \\
\hline CS & 1600.46 & $2.7 \mathrm{e}-6$ & 1600.46 & 1600.46 \\
\hline $\mathrm{ABC}$ & 1607.37 & 11.676 & 1620.60 & 1600.51 \\
\hline FA & 1617.34 & 10.746 & 1633.57 & 1600.47 \\
\hline PSO & 1609.13 & 8.231 & 1627.87 & 1600.60 \\
\hline SFL & 1602.06 & 1.519 & 1607.62 & 1600.67 \\
\hline $\mathrm{BFO}$ & 1604.28 & 3.1993 & 1611.35 & 1600.02 \\
\hline HS & 1610.10 & 9.415 & 1629.18 & 1600.58 \\
\hline GSA & 1612.24 & 6.567 & 1618.67 & 1600.56 \\
\hline GLSPSA & 1541.1 & $2.3 e-6$ & 1541.1 & 1541.1 \\
\hline
\end{tabular}

\section{Comments}

\section{- Global Minima Solution}

From cost analysis in table 3, proposed technique achieve minima cost solution 2000.039(\$hr) as compared to other techniques PS, DE, RCGA, BFGS, BCGA for IEEE 25 bus system. Further on, Table 6 showcase the cost result 7891.1 ( $\$ / \mathrm{hr})$ for six unit system and comparison is made with methods PSO, ABC, SFLA, CS, HS, BFO, GSA,FA. From result inference, we see that proposed technique gives minimum cost when compared with other techniques. Table 8 again reflects the cost $1541.1(\$ / \mathrm{hr})$ result for three unit system which is minimum as compared to reported techniques. Thus, proposed method GLSPSA has the strong capability of reaching global solution.

- Computational Power

From table 3, we see that the proposed technique takes less computational time (1.4 $\mathrm{sec})$ as compared to other techniques DE, BCGA, RCGA. This technique consumes less CPU memory as it is fast due to fast convergence from Figure 5,6. From table $5,7,8,9$-we observe that deviation of solution $(2.3 \mathrm{e}-6,0.000097)$ for this technique GLSPSA is less as compared to PSO, ABC, SFLA, CS, HS, BFO, GSA,FA for three and six unit system. Best, mean and worst cost with different set of iterations shows the computational efficiency of proposed method as they keep minima value as tested with other methods. 


\section{- Convergence Precision Capability}

From Figure. 5 and 6, convergence of solutions for iterations has been tested. It shows that, proposed methodology grows towards global solutions and it maintains the solution convergence for many runs. Table 7 and 9 shows that best, mean and worst solution $(1541.1,1541.1,1541.1)$ are close approximation of each other, means the convergence precision is high and avoid all the local minima solutions among feasible

solutions in search boundary as compared to PSO, ABC, SFLA, CS, HS, BFO, GSA, FA as these algorithms suffers from huge standard deviation among its best, mean, worst which shows lack of precision of convergence.

\section{- Exploration for Optimal Solution}

As the proposed method GLSPSA explores solution in random initial population of generated powers through search space. Table 7 and 9 shows that average cost $(7891.1,1541.1)$ for GLSPSA method is minimum when compared to PSO, ABC, SFLA, CS, HS, BFO, GSA,FA for three and six unit system. It means average of all the solutions in feasible search space with different iterations carries the optimality when fly through the space for solution search. Further on, best cost also shows the promise of efficient exploration capability in table 7 and 9.SD goes down as the proposed method converge to optimal solution means the direction and boundary of solution search is best one.

\section{Conclusion}

In present work, GLSPSA is proposed for solving APD problems. The efficiency and effectiveness of the proposed technique is benchmarked for different test cases consisting of three, five and six-generating units. The results of the GLSPSA compared with that of other intelligence algorithms.

Wide contrasting simulation results are observed with the other algorithms, it is concrete that GLSPSA gives better results than other algorithms. GLSPSA is simple to formulate and potential of reaching feasible global optimal solution.

Further on, the solution backs the robustness, accurate convergence and optimality of this optimization technique. From the results obtained it can be concluded that GLSPSA is a promising technique for solving complex non smooth optimization problems.

\section{Future Scope}

In future, the proposed technique can be effectively applied to solve convex and nonconvex constraints and complex EPD problems with incorporation of more objectives and constraints

\section{References}

[1] S. Duman, U. Güvenç1, N. Yörükeren "Gravitational Search Algorithm for Economic Dispatch with Valve-Point Effects", International Review of Electrical Engineering (I.R.E.E.), Vol. 5, No. 6,pp.28902895,2010 .

[2] Wood, A.J and Wollenberg B.F., "Power generation, operation and control",2nd ed.,John Wiley, New York,1996.

[3] Zhu, J. , "Optimization of Power System Operation”, IEEE Press on Power Engineering, Wiley,2009.

[4] Navpreet Singh Tung and Sandeep Chakravorty ,"Active Power Dispatch Planning using Differential Evolution", Journal of Industrial and Intelligent Information Vol. 2, No. 3,pp 200-204,2014.

[5] Navpreet Singh Tung and Sandeep Chakravorty, "Active Power Dispatch Planning using Pattern Search”, International Journal of Electrical Energy, Vol. 2, No. 3(2014), pp. 216-220,2014.

[6] Hamid Boujeboudja et al ,"Economic Dispatch Solution using A real coded genetic Algorithm" ,Acta Electro-technica et Informatica(2005) No. 4, Vol. 5,pp.1-5,2005.

[7] Vikram Kumar Kamboj, S.K Bath ,"Mathematical Formulation of Multi Area Unit Commitment Problem", International Journal of Power System Operation and Energy Management, Volume-3, Issue2,2013 . 
[8] Algorithm", IOSR Journal of Electrical and Electronics Engineering (IOSRJEEE), Volume 1, Issue 4, pp. 09-26,2012.

[9] Vikram Singh et al, "Short Term Unit Commitment and Economic Load Dispatch Problem of thermal Electric Power System Using Particle Swarm Optimization", International Journal of Power System Optimization, 09 pp.10-005,2014.

[10] Vikram Kumar Kamboj, S.K Bath, "Scope of Biogeography Based Optimization for Economic Load Dispatch and Multi-Objective Unit Commitment Problem”, International Journal of Energy Optimization and Engineering Vol.3.Issue.4, pp.34-54,2014.

[11] Adriane B. S. Serapião," Cuckoo Search for Solving Economic Dispatch Load Problem”, Intelligent Control and Automation, Vol.4, pp. 385-390,2013.

[12] Mirjalili S, Mohd Hashim S Z, "A new Hybrid PSOGSA Algorithim for Function Optimization”, IEEE International Conference on Computer Information and application (ICCIA 2010), China ,pp. 374377,2010 .

[13] a Local Search Algorithm for Economic Emission Load Dispatch Problem”, Applied Mathematics,Vol. 2, pp.890-898,2011.

[14] Manisha Sharma et al," Multi-area economic dispatch with tie-line constraints employing evolutionary approach", International Journal of Engineering, Science and Technology, Vol. 2, No. 3, pp. 132$149,2010$.

[15] Dr.T.Govindaraj ,'Optimal Economic Dispatch for Power Generation Using Genetic Algorithm”, International Journal of Innovative Research In Electrical, Electronics, Instrumentation and Control Engineering Vol. 2, Issue 1, January 2014

[16] Priyanka Roy, "Modified Shuffled Frog Leaping Algorithm for Solving Economic Load Dispatch Problem" Energy and Power Engineering, vol.3, pp.551-556,2011.

\section{Authors}

Navpreet Singh Tung is serving as an Assistant Professor in Department of Electrical Engineering, Bhutta Group of Institutions. India. He holds his B-Tech in Instrumentation and Control Engineering from National Institute of Technology, Jalandhar. He obtained his M-Tech in Electrical Engineering with specialization in Power System from Lovely Professional University. He is a member of reviewer board of International Journals. He authored and co-authored many papers in leading international proceedings and journals in Power System. His area of interest is Power System Planning, Power System Optimization.

Prof.(Dr.)Sandeep Chakravorty is serving as a Dean and Professor in Department of Electrical Engineering, Baddi University, India.He did his BE in Department of Electrical and Electronics Engineering, Sikkim Manipal Institute of Technology, Sikkim and ME in Software Engineering from Birla Institute of Technology, Mesra. Ranchi.He obtained his PHD in Power System Planning from Sikkim Manipal University. He served in different capacities in Sikkim Manipal University, Lovely Professional University. He has a long stint of teaching and research career in Electrical Engineering. He authored and coauthored many research papers in the area of Power system in leading International Journals and Conferences. His area of expertise is Power System Planning, Power system Optimization and application of artificial intelligence in Power System. 
International Journal of Grid and Distributed Computing Vol.9, No.5 (2016) 